\title{
Two-way least-incision transformable end-effector forceps for robot-assisted surgery
}

\author{
Yuki Yamamoto ${ }^{1}$ and Ryoichi Nakamura ${ }^{*}$
}

\begin{abstract}
In laparoscopic surgery, smaller incisions produce good clinical results for patients. Therefore, there is a great need to develop thinner instruments for use in laparoscopic surgery. However, thinner instruments have smaller end effectors that limit the functionality of the instruments. To address this problem, we previously developed a new type of forceps that uses a least-incision transformable end-effector (LITE) mechanism for graspers of two sizes (i.e., a two-way LITE mechanism), which decreases the instrument exchange frequency and the total procedure time during surgery. Robot-assisted surgery is currently receiving considerable attention. However, instrument exchange during robot-assisted surgery is complicated. The purpose of this study was to develop a new two-way LITE forceps that would be suitable for use in robot-assisted surgery and laparoscopic surgery by being attached to a handpiece to reduce the time and difficulty associated with the instrument exchange during robot-assisted surgery. The experimental results show that the proposed forceps can reduce the total instrument exchange time in a simulated robot-assisted surgery environment, generate sufficient grasping forces to serve as a laparoscopic grasper, and perform switching between its small-grasper and large-grasper modes when operated with one hand using the handpiece. An in vivo experiment was performed on a pig under simulated surgery conditions to evaluate the usefulness of the prototype developed in this study. When operated with a motor drive, the forceps was able to grasp the liver using its large grasper.
\end{abstract}

Keywords: Laparoscopic surgery; Forceps; Robot-assisted surgery

\section{Background}

Laparoscopic surgery is performed by inserting a camera and an instrument through small incisions in the body cavity. In laparoscopic surgery, smaller incisions produce less physical stress for patients [1]. Therefore, studies are being carried out to develop thinner surgical instruments [2]. However, as instruments become thinner, the sizes of the end effectors also decrease. Consequently, grasping an organ becomes difficult because of the decrease in grasper size [3]. To address this problem, new instruments that transform into large graspers inside the body cavity have been developed $[4,5]$. In laparoscopic surgeries that require the use of various instruments, exchanging instruments is a complicated task that consumes $10-30 \%$ of the total procedure time [6]. Therefore, a single instrument

\footnotetext{
* Correspondence: ryoichin@faculty.chiba-u.jp

${ }^{2}$ Center for Frontier Medical Engineering, Chiba University, Chiba, Japan

Full list of author information is available at the end of the article
}

that has multiple functions can reduce the amount of instrument exchange required and thereby reduce the total procedure time [7].

In a previous study, we developed a forceps with a least-incision transformable end-effector (LITE) mechanism for operating graspers of two sizes (i.e., a two-way LITE mechanism) that switches its end effectors to change its size inside the body cavity [8]. The forceps is inserted into the body through a small incision and is used as a large end effector. The forceps decreases the instrument exchange frequency and the total procedure time during surgery.

Robot-assisted surgery is currently receiving considerable attention because it has many advantages, such as improved operability and improved field of vision during laparoscopic procedures. However, quick exchange of surgical instruments is difficult because an assistant performs the instrument exchange on the instructions of

\section{曾}

(c) 2015 Yamamoto and Nakamura; licensee Springer. This is an open access article distributed under the terms of the Creative Commons Attribution License (http://creativecommons.org/licenses/by/4.0), which permits unrestricted use, distribution, and reproduction in any medium, provided the original work is properly credited. 
the surgeon, and there are many processes to be followed in exchanging an instrument. The use of a two-way LITE mechanism with surgical robots can reduce the difficulty and time associated with instrument exchange. Furthermore, during robot-assisted surgery, the instruments used in laparoscopic surgery must be prepared because the surgeon may need to switch to performing laparoscopic surgery in an emergency. Therefore, hand-held instruments that can be mounted on a surgical robot have many advantages, such as space and cost savings.

The purpose of this study was to develop a new twoway LITE forceps that can be attached to a surgical robot and used for laparoscopic surgery by attaching a handpiece to the forceps. In other words, we adapted the previously developed hand-held two-way LITE forceps to a surgical robot.

\section{Methods}

\section{Two-way LITE forceps for robot-assisted surgery}

The two-way LITE forceps for robot-assisted surgery was developed with reference to a surgical instrument of da Vinci ${ }^{\circledR}$ surgical system [9] which has been used for many clinical applications [10].The working of the end effector of the da Vinci ${ }^{\oplus}$ instrument is based on the rotation of four disks connected to the side that is attached to the surgical robot. The two-way LITE mechanism performs the movements shown in Figure 1 by the operation of three rods associated with a 5-mm grasper, a 10-mm grasper, and a transformation. The end effector works by performing a linear-slide motion of $3 \mathrm{~mm}$ for the $5-\mathrm{mm}$ or $10-\mathrm{mm}$ grasper rod or $5 \mathrm{~mm}$ for the transformation rod. We developed a forceps in which the end effector works by the rotation of three disks (Figure 2). Inside the forceps, the rotation of the disks is converted into a linear-slide motion through a slider-crank mechanism.

\section{Handpiece}

When the interface of the conventional two-way LITE forceps is used, it is difficult to switch modes using one hand because two switches need to be operated. The first switch, on the back side of the forceps, is used to move the transformation rod. The second switch, on the lateral side of the forceps, is used to exchange the grasping rod. Therefore, we developed a handpiece that can be attached to the forceps and used in laparoscopic

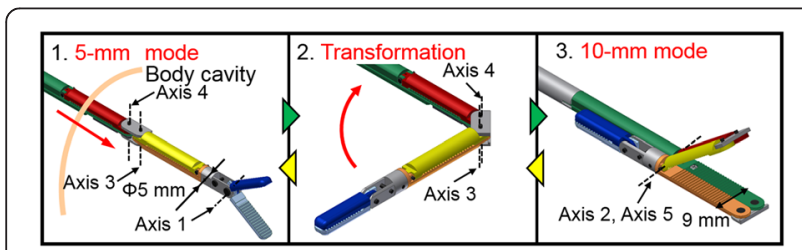

Figure 1 Transformation procedure.

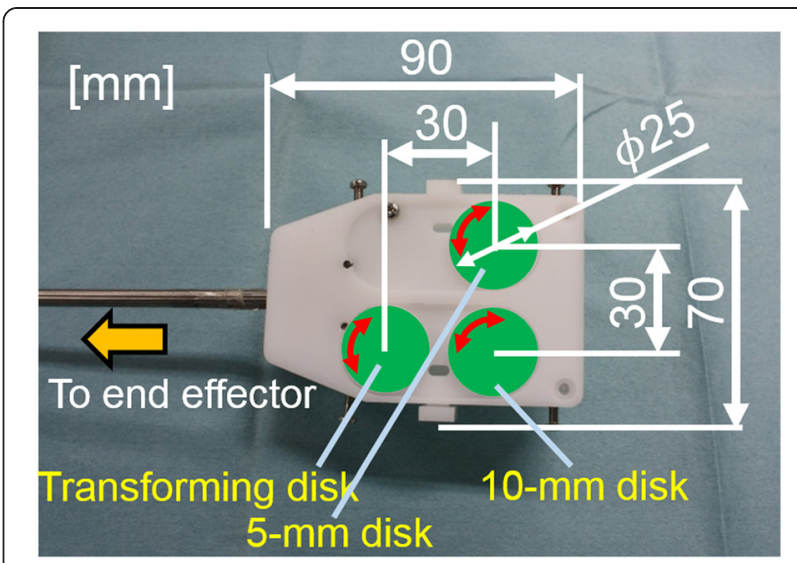

Figure 2 Two-way LITE forceps.

surgery. The handpiece facilitates mode switching by the operation of only one switch (Figure 3). The three disks of the forceps can be rotated by operating the handle and switch of the handpiece.

\section{Mode-switching mechanism}

The transformation disk and the switch form a sliderlink mechanism (Figure 4(a)). The transformation disk rotates when the switch is operated, and the end effector of the forceps undergoes transformation. In addition, the handle operation rotates the grasping disk through a shaft (Figure 4(b)). When the switch is operated, the shaft shifts perpendicular to the direction of the switch movement, and the grasping disk that is operated by the handle is switched (Figure 4(c)).

\section{Prototype}

The prototype of the handpiece that is attached to the forceps is shown in Figure 5. It was confirmed that grasping and transformation can be achieved by operating the handle and switch.

\section{Evaluation experiment}

\section{Instrument exchange time measurement experiment}

For comparison purposes, we measured the modeswitching time required by the proposed forceps when fitted with a motor drive, and we measured the instrument exchange time during robot-assisted surgery. The

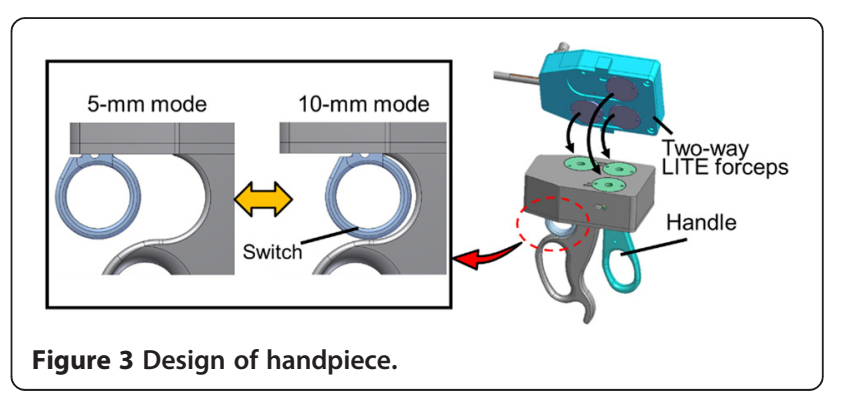


a

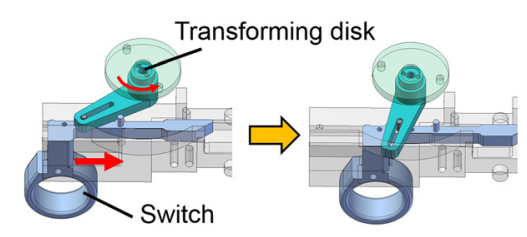

b

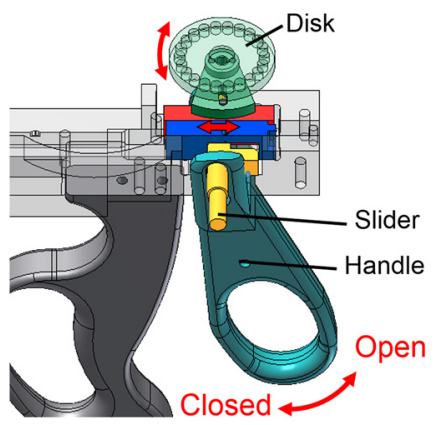

C

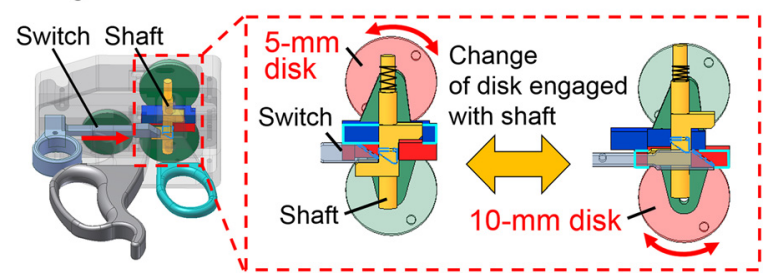

Figure 4 Handpiece mechanisms: (a) Transformation mechanism (b) Grasping operation mechanism (c) Change of disk engaged with handle.

forceps was attached to the experimental device, in which three disks were rotated using the motor. The rotary speed produced allowed the experimental device to perform transformation in $6 \mathrm{~s}$. An environment that mimicked a robot-assisted surgery environment was created as described in a previous study [11]. Five subjects (non-specialists) performed instrument exchanges six times in this environment, and the exchange times were measured. The instrument exchange process is as follows. Process 1 involves pulling the instrument out of a trocar. Process 2 is exchanging the instrument. Process 3 is attaching the instrument to the surgical robot. Process 4 is inserting the instrument into the trocar. The experimental processes were explained to the subjects beforehand, and

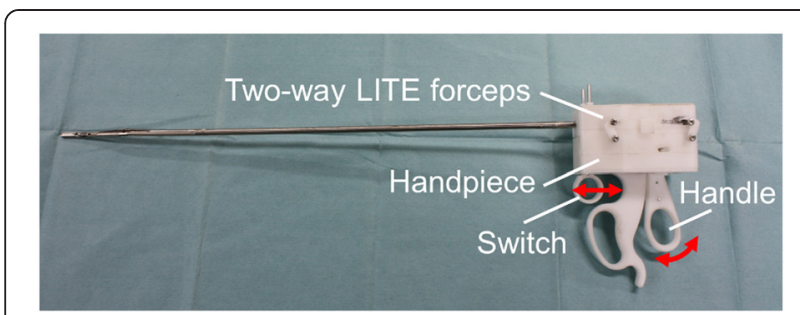

Figure 5 Handpiece equipped with two-way LITE forceps. they started the experiment after practicing the process under the same conditions as those of the experiment.

\section{Grasping force measurement experiment}

The grasping forces generated by the forceps using the motor drive and the handpiece were measured. Both experimental systems are shown in Figure 6. In the measurement of the grasping force achieved using the motor drive, the disk of the forceps was given suitable rotation. Grasping was maintained for four seconds, and the grasping force was measured eight times. The motor used was a stepping motor (CRK564PMAPB: Oriental Motor Co., Ltd., maximum torque: $0.78 \mathrm{Nm}$, basic step angle: $\left.0.36^{\circ}\right)$. In the measurement of the grasping force achieved using the handpiece, weights ranging from 0 to $30 \mathrm{~N}$ were hung on the handle in increments of $2 \mathrm{~N}$.

\section{In vivo experiment}

Transformation and grasping of the forceps using the motor drive and the handpiece were tested in an experiment performed on a pig under simulated laparoscopic surgery conditions. We tested the forceps to ensure that it functioned normally in vivo, and we used it to lift the liver, which is the heaviest organ in the body.

\section{Mode-switching time measurement experiment using the handpiece}

We measured the mode-switching time required by the forceps when using the handpiece and by the conventional two-way LITE forceps to compare their operability. The transformation time from the $5-\mathrm{mm}$ mode closed state to the $10-\mathrm{mm}$ mode open state and the removal time from the $10-\mathrm{mm}$ mode closed state to the 5 -mm mode open state were measured. Five subjects used a laparoscopic surgery trainer device (MATT Trainer) to switch between the $5-\mathrm{mm}$ and $10-\mathrm{mm}$ modes, and the mode-switching time required by each of these subjects was measured five times. The experimental processes were explained to the subjects beforehand, and they started the experiment after

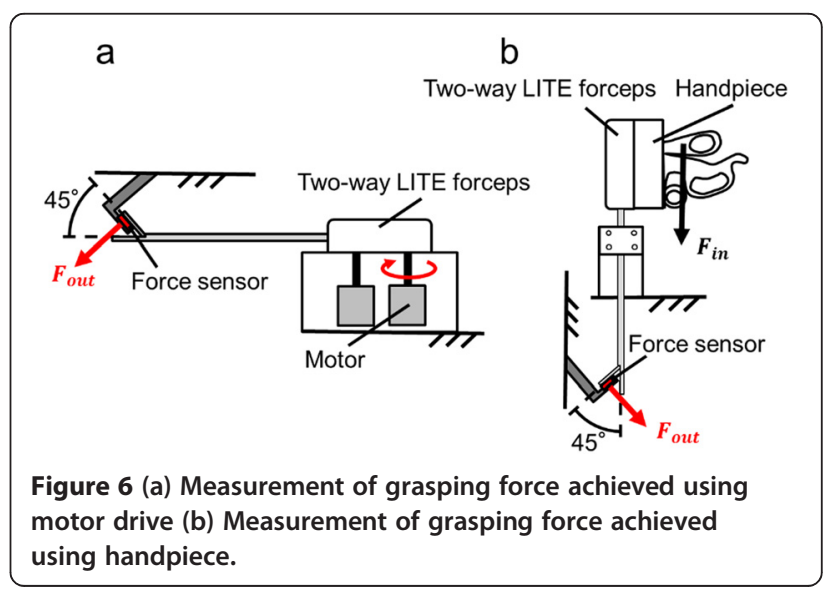


practicing the process under the same conditions as those of the experiment.

\section{Results and discussion}

\section{Results of instrument exchange time measurement} experiment

The instrument exchange time was measured in the robot-assisted surgery environment and was found to be $19.5 \pm 6.3 \mathrm{~s}$. The motor-driven forceps was able to perform switching in $6 \mathrm{~s}$. The mode-switching time for the motor-driven forceps can be reduced because of the rotary speed of the motor. We infer that the instrument exchange time can be reduced using the two-way LITE forceps in robot-assisted surgery. The number of times that instruments need to be exchanged during robotassisted surgery will also be reduced because the twoway LITE forceps can switch the grasping mode without complicated instrument exchange processes.

\section{Results of grasping force measurement experiment}

The grasping force achieved by the forceps using the motor drive was $4.86 \pm 0.17 \mathrm{~N}$ in the 5 -mm mode and $5.51 \pm 0.03 \mathrm{~N}$ in the $10-\mathrm{mm}$ mode. These values are averages calculated for grasps maintained for $4 \mathrm{~s}$. The results of the measurement of the grasping force achieved using the handpiece are shown in Figure 7. A previous study concluded that the pinch force necessary to avoid slippage is more than $3.3 \mathrm{~N}$ [12]. Because the forceps was able to produce grasping forces of more than $3.3 \mathrm{~N}$ with both the motor drive and the handpiece, we conclude that the forceps produces sufficient grasping force for it to be used in practical applications. The disk rotation was adjusted to avoid damage to the two-way LITE mechanism during the motor drive experiment. Changing the material and improving the structure of the forceps will increase the grasp force that the forceps is able to produce.

The maximum grasp force generated by the forceps using the handpiece was approximately $3.8 \mathrm{~N}$ in the $10-\mathrm{mm}$ mode. Because the disk rotation is limited by

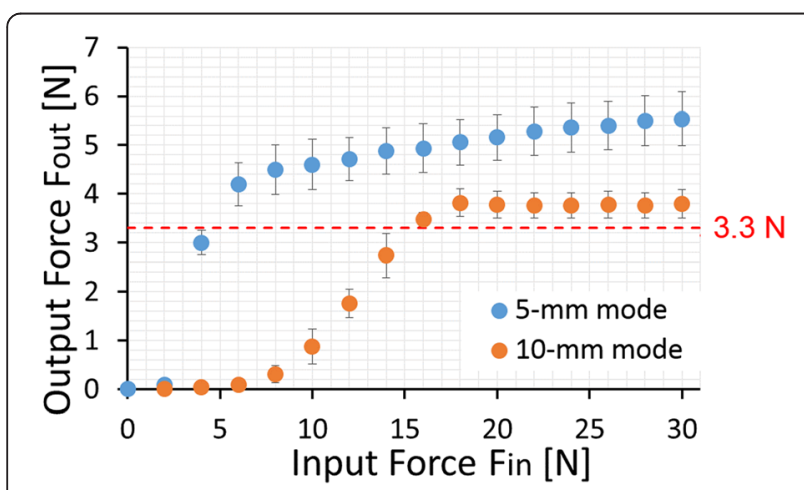

Figure 7 Results of measurement of grasping force achieved using handpiece. the operating range of the handle, the forceps cannot produce a grasping force greater than this maximum value using the handpiece. When using the motor drive, the forceps produced a greater grasping force because of the larger rotation possible. The rotation of the disks can be increased by improving the grasping operation mechanism of the handpiece.

\section{Results of in vivo experiment}

The forceps could not achieve $180^{\circ}$ flexural transformation using the motor drive (Figure 8(a)), but the forceps was able to perform grasping in the $5-\mathrm{mm}$ and $10-\mathrm{mm}$ modes and perform mode switching. Furthermore, the motor-driven forceps was able to grasp the liver in the $10-\mathrm{mm}$ mode (Figure $8(\mathrm{~b})$ ). The problem of $180^{\circ}$ flexural transformation not being achieved also occurred when the conventional two-way LITE forceps was used. Because of the high elasticity of the super-elastic alloy utilized in the flexural joint, the grasping rod did not achieve proper transformation in the $5-\mathrm{mm}$ mode. This problem will be addressed in the future by using a super-elastic alloy with a lower elasticity.

Although the transformation achieved when using the handpiece was not as good as that achieved when using the motor drive, grasping was successful in the $5-\mathrm{mm}$ and $10-\mathrm{mm}$ modes. However, the rod did not switch when the $10-\mathrm{mm}$ mode was switched to the $5-\mathrm{mm}$ mode. Therefore, even though the end effector was extended, the operation of the 10 - $\mathrm{mm}$ grasper was not ideal (Figure 8 (c)), and the 5-mm grasper could not be operated. One of reasons for this was that the two-way LITE mechanism tilted about the longitudinal axis and was fixed to the interface. Therefore, it is believed that the linear-slide motion of the 5-mm rod is not performed smoothly and that the disk of the handpiece does not move to the most appropriate position for switching the mode. This problem will be addressed in the future by including a spring that will assist the rotation of the $5-\mathrm{mm}$ disk inside the handpiece.

\section{Results of the mode-switching time measurement experiment using the handpiece}

The results of the mode-switching time measurement experiment conducted using the handpiece are shown in Figure 9. The subjects operated the forceps with their left hands only. When using the conventional two-way LITE forceps, the subjects used both hands, and the switching times were measured [8].

In both modes, the mode-switching time of the forceps when using the handpiece was significantly different from the mode-switching time of the conventional two-way LITE forceps. The proposed forceps required more time to switch to the 10-mm mode than the conventional twoway LITE forceps. However, for switching to the 5-mm 

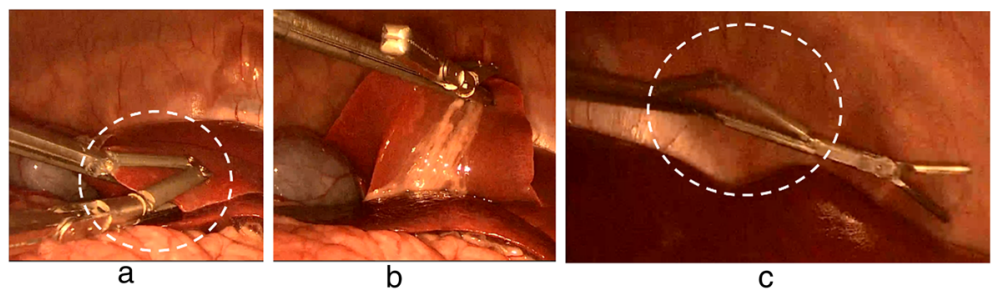

Figure 8 In vivo experiment results: (a) Incomplete transformation (b) Grasped liver (c) Incomplete switching.

mode, it required approximately half the time required by the conventional two-way LITE forceps because the switching could be accomplished with only one switch operation. The forceps must open the 5 -mm grasper before the transformation for the $10-\mathrm{mm}$ mode. The reason for this is that the forceps must transform the end effector after the $5-\mathrm{mm}$ disk is rotated for the path length of the 5 - $\mathrm{mm}$ rod in the forceps to change. This operation adds to the handle operation processes, and hence, the time required for switching in the $10-\mathrm{mm}$ mode increases. This problem will be addressed in the future by improving the switch mechanism by which the transformation is carried out after the operation rod is switched.

It is believed that using the handpiece, the forceps can also be used as an auxiliary forceps because mode switching can be performed with the left hand only. The auxiliary forceps can be operated with the left hand to assist the main forceps that can be operated with the right hand.

\section{Conclusions}

This study focused on reducing the time and difficulty involved in instrument exchange during robot-assisted surgery and resulted in the development of a two-way LITE forceps for use with a surgical robot system. This forceps can be used in laparoscopic surgery by attaching it to a handpiece. Evaluation and in vivo experiments were performed to test the forceps. The forceps reduced the instrument exchange time in a robot-assisted surgery environment and was able to generate sufficient grasping force. Furthermore, the forceps performed mode switching in a laparoscopic surgery environment in the same

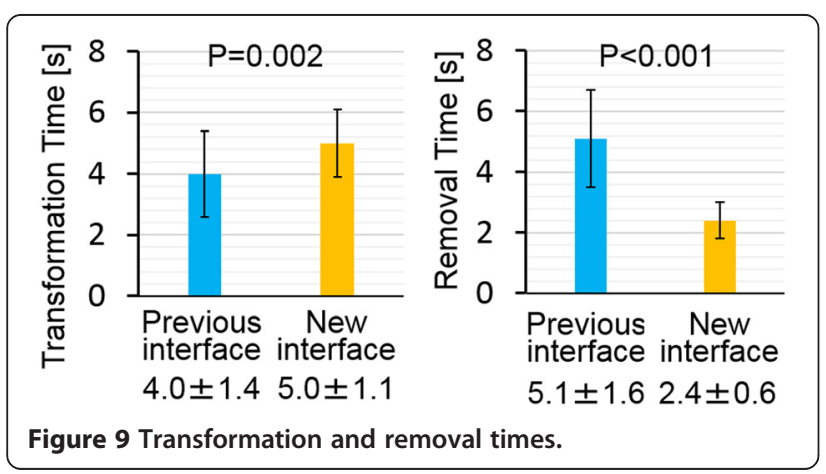

amount of time as the conventional two-way LITE forceps. In addition, the proposed forceps could be operated using one hand when performing mode switching. The grasping operation was performed in vivo. In addition, using the motor drive, the forceps could grasp an organ using a large grasper. These results suggest that the new forceps developed in this study can be used as an auxiliary forceps to assist the forceps held in the dominant hand.

\section{Abbreviation}

LITE: Least-incision transformable end effector.

\section{Competing interests}

The authors declare that they have no competing interests.

\section{Authors' contributions}

YY developed and evaluated the devices and drafted the manuscript. RN conceived of the study, participated in its design and coordination, and helped in drafting the manuscript. Both authors read and approved the final manuscript.

\section{Acknowledgments}

Authors would like to thank Profs. Hiroshi Kawahira and Tatsuo Igarashi for their comments and support for the clinical aspects of this research. This study is partly supported by Grant-in-Aid for Young Scientists (A) (23680056) by MEXT and Chiba Univ. VBL Project grant.

\section{Author details}

${ }^{1}$ Graduate School of Engineering, Chiba University, Chiba, Japan. ${ }^{2}$ Center for Frontier Medical Engineering, Chiba University, Chiba, Japan.

Received: 7 October 2014 Accepted: 27 January 2015

Published online: 08 February 2015

\section{References}

1. Tagaya N, Kubota K (2012) Reevaluation of needlescopic surgery. Surg Endosc 26(1):137-143

2. Yamashita H, Matsumiya K, Masamune K, Liao H, Chiba T, Dohi T (2006) Two-DOFs bending forceps manipulator of 3.5-mm diameter for intrauterine fetus surgery: feasibility evaluation. Int J Comput Assist Radiol Surg 1:218-220

3. Ohdaira T, Tsutsumi N, Xu H, Mori M, Uemura M, leiri S et al (2011) Ultra-minimally invasive local immune cell therapy and regenerative therapy by multi-piercing surgery for abdominal solid tumor: therapeutic simulation by natural orifice translumenal endoscopic surgery-assisted needlescopic surgery using 3-mm diameter. J Hepatobiliary Pancreat Sci 18:499-505

4. Nakaji H, Nakamura R (2011) Miniaturization of least-incision transformable end-effector mechanism for forceps for endoscopic surgery. Int J CARS 6(suppl1):241-242

5. Abe T, Nakaji H, Nakamura R (2012) Development of the bending mechanism for forceps with least-incision transformable end-effector mechanism for endoscopic surgery: Development of the operating interface and in vivo experiment. In: 4th IEEE RAS \& EMBS International Conference on Biomedical Robotics and Biomechatronics (BioRob), Rome, IEEE; 24-27 
June 2012, pp. 919-923. http://ieeexplore.ieee.org/xpl/login.jsp?tp=\&arnumber $=6290934 \&$ url=http\%3A\%2F\%2Fieeexplore.ieee.org\%2Fxpls\%2Fabs_all.jsp \%3Farnumber\%3D6290934

6. Melzer A (1996) Endoscopic instruments: conventional and intelligent. In: Gossot D, Hunter JG (eds) Tooli J. Endosurgery, Churchill Livingstone, New York, NY

7. Frecker MI, Schadler J, Haluck RS, Culkar K, Dziedzic R (2005) Laparoscopic multifunctional instruments: design and testing of initial prototypes. JSLS 9(1):105-112

8. Abe T, Nakamura R (2013) Two-way LITE mechanism: Least-incision transformable end-effector (LITE) mechanism for two size graspers on endoscopic forceps. Procedia CIRP 5:137-140

9. Guthart GS, Salisbury Jr JK (2000) The intuitive telesurgery system: overview and application. In: Proceedings of the IEEE International Conference on Robotics and Automation. IEEE, Pp. 618-621. http://ieeexplore.ieee.org/xpl/ login.jsp?tp=\&arnumber=844121\&url=http\%3A\%2F\%2Fieeexplore.ieee.org\% 2Fxpls\%2Fabs_all.jsp\%3Farnumber\%3D844121

10. Kenngott HG, Fischer L, Nickel F, Rom J, Rassweiler J, Müller-Stich BP (2012) Status of robotic assistance-a less traumatic and more accurate minimally invasive surgery? Langenbecks Arch Surg 397(3):333-341

11. Higuchi TT, Gettman MT (2011) Robotic instrumentation, personnel and operating room setup, In: Atlas of Robotic Urologic Surgery. Humana Press, 15-30. http://link.springer.com/chapter/10.1007\%2F978-160761-026-7_2

12. Heijinsdijkp EAM, Pasdeloup A, Dankelman J, Gouma DJ (2004) The optimal mechanical efficiency of laparoscopic forceps. Surg Endosc 18:1766-1770

\section{Submit your manuscript to a SpringerOpen ${ }^{\circ}$ journal and benefit from:}

- Convenient online submission

- Rigorous peer review

- Immediate publication on acceptance

- Open access: articles freely available online

- High visibility within the field

- Retaining the copyright to your article 